\title{
The mere exposure effect for visual image
}

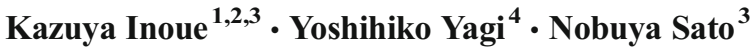

Published online: 30 August 2017

(C) Psychonomic Society, Inc. 2017

\begin{abstract}
Mere exposure effect refers to a phenomenon in which repeated stimuli are evaluated more positively than novel stimuli. We investigated whether this effect occurs for internally generated visual representations (i.e., visual images). In an exposure phase, a $5 \times 5$ dot array was presented, and a pair of dots corresponding to the neighboring vertices of an invisible polygon was sequentially flashed (in red), creating an invisible polygon. In Experiments 1, 2, and 4, participants visualized and memorized the shapes of invisible polygons based on different sequences of flashed dots, whereas in Experiment 3, participants only memorized positions of these dots. In a subsequent rating phase, participants visualized the shape of the invisible polygon from allocations of numerical characters on its vertices, and then rated their preference for invisible polygons (Experiments 1, 2, and 3). In contrast, in Experiment 4, participants rated the preference for visible polygons. Results showed that the mere exposure effect appeared only when participants visualized the shape of invisible polygons in both the exposure and rating phases (Experiments 1 and 2), suggesting that the mere exposure effect occurred for internalized visual images. This implies that the sensory inputs from repeated stimuli play a minor role in the mere exposure effect. Absence of the mere exposure effect in Experiment 4
\end{abstract}

Kazuya Inoue

kazuyainoue@k-inoue.info

1 National Institute of Advanced Industrial Science and Technology, Tsukuba, Japan

2 Faculty of Engineering, Information, and Systems, University of Tsukuba, 1-1-1 Tennodai, Tsukuba, Ibaraki 305-8573, Japan

3 Kwansei Gakuin University, Nishinomiya, Japan

4 Rissho University, Shinagawa, Japan suggests that the consistency of processing between exposure and rating phases plays an important role in the mere exposure effect.

Keywords Mere exposure effect · Visual image $\cdot$ Perceptual fluency

The mere exposure effect is a phenomenon where repeatedly presented stimuli are evaluated more positively than novel stimuli. Since the seminal study of Zajonc (1968), more than 200 articles have been published on the mere exposure effect (Monahan, Murphy, \& Zajonc, 2000). Early studies of this effect focused upon identifying the relationship between exposure and affect from the viewpoint of stimulus variables and presentation variables (see Bornstein, 1989). One of the most important findings from these studies concerned the impact of the presentation duration of stimuli (Bornstein \& D'Agostino, 1992; Kunst-Wilson \& Zajonc, 1980; Mandler, Nakamura, \& Van Zandt, 1987; Seamon, Brody, \& Kauff, 1983). Specifically, it was shown that the mere exposure effect could occur even for brief stimuli, presented below a recognition threshold, suggesting that the conscious awareness of repeated stimuli is not a necessary condition for the mere exposure effect. Rather, a meta-analytic study revealed that stimuli perceived without awareness showed a considerably larger effect compared with stimuli that are consciously perceived (Bornstein, 1989). In fact, a subsequent experimental study provided direct support for this meta-analytic finding (Bornstein \& D'Agostino, 1992).

Based on these early findings, several accounts have been proposed to explain the mechanism underlying the mere exposure effect (Bornstein \& D'Agostino, 1992; Kunst-Wilson $\&$ Zajonc, 1980; Mandler et al., 1987). Of these accounts, the most widely accepted is the attribution of perceptual fluency 
theory (Bornstein \& D'Agostino, 1992, 1994). This account postulates that perceptual fluency (i.e., the ease of processing stimuli) is enhanced by repeated exposure to neutral stimuli. In turn, enhanced perceptual fluency can be misattributed to certain affective states, such as attributing "liking" to more familiar stimuli.

Perceptual fluency also plays an important role in the hedonic fluency theory (Winkielman \& Cacioppo, 2001). However, this view assumes that enhanced perceptual fluency causes the mere exposure effect without a misattributional process because perceptual fluency itself is deemed to have positive affective value. Indeed, it is widely accepted that enhanced perceptual fluency increases positive affect (Reber, Schwarz, \& Winkielman, 2004; Topolinski \& Strack, 2009). However, the mere exposure effect cannot be exclusively explained by the enhanced perceptual fluency. In fact, Monahan et al. (2000) reported that repeated presentation of neutral stimuli (in an exposure phase) increased positive affect to other unexposed stimuli compared with a single presentation of the neutral stimuli (in a following rating phase). This is inconsistent with the perceptual fluency account because there is no plausible reason to assume that unexposed stimuli should be perceived fluently. Based on the classical conditioning theory, Monahan et al. (2000) argued that reduced alertness and tension due to repeated exposure are involved in the mere exposure effect (see Zajonc, 2001). In sum, there is no conclusive account that can comprehensively explain the existing findings.

In contrast to the most earlier studies dealing with the stimulus variables, another important line of research, which began in the mid-1990s, focused on a cognitive factor involving internal representations that underlie the mere exposure effect. To this end, some studies have investigated the generalization of the mere exposure effect by changing the properties of stimuli (e.g., between exposure and a later rating phase.) For example, the mere exposure effect based on repeated presentations of three-dimensional figures (during an exposure phase) was not impaired by reflection or size transformation of these figures in rating phase (Seamon et al., 1997). Similarly, the mere exposure effect for face stimuli was unaffected by the change in the horizontal angle of repeated faces (Kramer \& Parkinson, 2005). The generalization of the mere exposure effect also occurs for averaged composites of repeatedly presented faces (Rhodes, Halberstadt, \& Brajkovich, 2001). Together, such findings demonstrate that the mere exposure effect could occur for unexposed but similar stimuli. In turn, this implies that the internal representation underlying the mere exposure effect is insensitive to a subtle change of repeated stimuli.

The importance of an internal representation in the mere exposure effect was directly supported by a study manipulating perceptual sets for exposed stimuli (Craver-Lemley \& Bornstein, 2006). In this study, participants perceiving an ambiguous duck-rabbit figure as a duck in the exposure phase demonstrated the mere exposure effect by later preferring the figure of a disambiguated duck, whereas participants perceiving it as a rabbit demonstrated this effect in their preferences for the figure of a disambiguated rabbit. A similar finding was also reported by a study investigating the effect of selective attention on the mere exposure effect (Yagi, Ikoma, \& Kikuchi, 2009); here, participants were shown a composite figure consisting of a green polygon and a red polygon and were asked to selectively attend to one of the polygons while ignoring the other. The mere exposure effect was found in a later preference for the attended polygon, not for the ignored or composite polygons. Taken together, these studies suggest that the mere exposure effect occurs for the internal representation of repeated stimuli modulated by top-down factors, such as perceptual set and selective attention, but not for the sensory input from the repeated stimuli per se.

Although previous studies have revealed the importance of the internal representation of repeated stimuli in the mere exposure effect, it remains unclear whether the sensory input from repeated stimuli (i.e., physical shape) plays a significant role in the mere exposure effect. Given the importance of the top-down factors (Craver-Lemley \& Bornstein, 2006; Yagi et al., 2009), it is possible that sensory input from repeated stimuli are not a necessary factor to the mere exposure effect. That is, the mere exposure effect could occur even though the stimuli presented in the rating phase are dissimilar in physical shape to those presented in the exposure phase as long as the internal representation of shape used in the exposure phase is accessed in the later rating phase. To the best of our knowledge, this possibility remains to be tested. In most previous studies on the mere exposure effect, the to-be-rated stimuli were similar to or contained within the stimuli in the preceding exposure phase (Craver-Lemley \& Bornstein, 2006; Kramer \& Parkinson, 2005; Seamon et al., 1997; Yagi et al., 2009).

Therefore, the present study was conducted to clarify whether sensory input from repeated stimuli is a necessary factor for the mere exposure effect. In other words, we investigated whether the construction of internal representations is critically important for instantiating the mere exposure effect. To this end, we devised an experimental paradigm where shape representations constructed in the exposure phase could be accessed in the rating phase although the sensory inputs from repeated stimuli were dissimilar to those in the rating phase (see Figs. 1 and 2). In the exposure phase, a $5 \times 5$ dot array, superimposed on an invisible polygon, was presented. Two dots of the array, which were on adjacent vertices of an invisible polygon, were sequentially flashed in red, indicating the shape of the invisible polygon. Participants were required to visualize the shape of this invisible polygon by mentally connecting flashed dots with a line. In the subsequent rating phase, consecutive numerical characters from 1 to 5 were presented on the vertices of the invisible polygon in a counterclockwise direction. Participants 
repeated two times

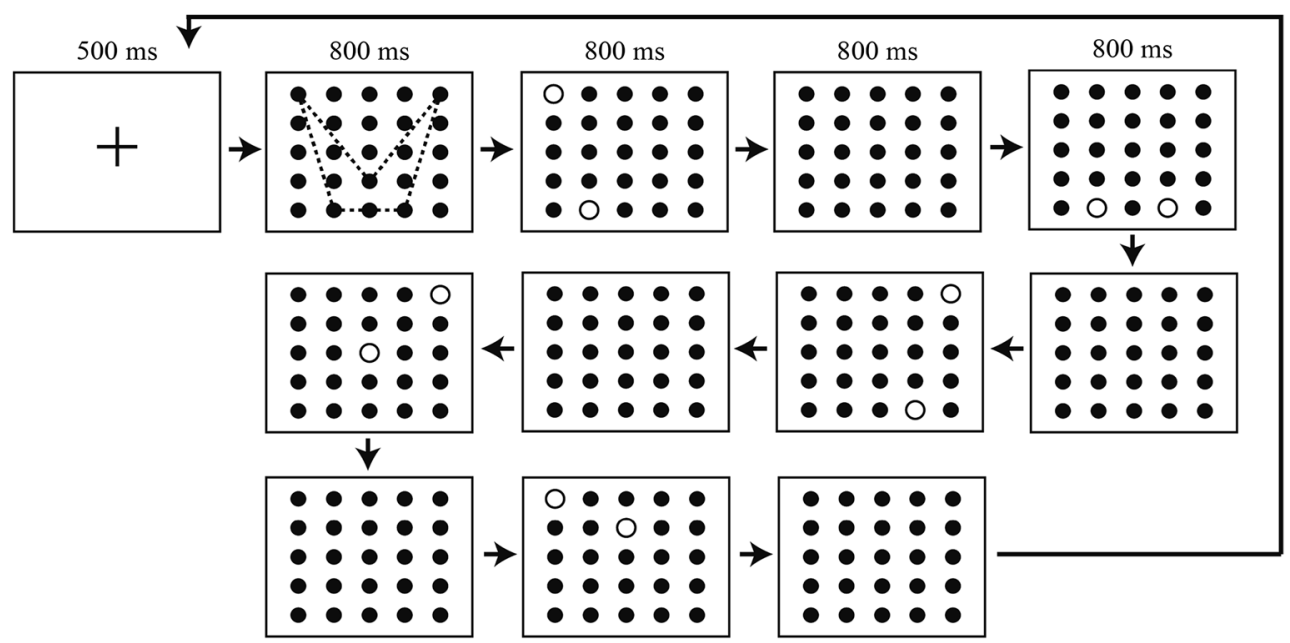

Fig. 1 Schematic illustration of a trial in an exposure phase. Polygon shown in dashed line was not actually presented (i.e., an invisible polygon). A pair of dots on the vertices of the invisible polygon was flashed in red for $800 \mathrm{~ms}$ and returned to black for $800 \mathrm{~ms}$. Another

should visualize the invisible polygon by mentally connecting the numbers in ascending order and rate the preference for the shape of the visualized polygon. The aim of Experiment 1 was to investigate whether the mere exposure effect is observed with this experimental paradigm. If the sensory input from repeated stimuli is not a requisite factor for the mere exposure effect, then this effect should be evident in this paradigm. In addition, such an outcome would provide direct evidence that internal representation (in this study, visual image) of repeated stimuli plays a critical role in the mere exposure effect, thus offering corroboration of previous findings (Craver-Lemley \& Bornstein, 2006; Yagi et al., 2009).

Experiments 2 and 3 were conducted to confirm findings of Experiment 1. In Experiment 1, the order of flashed dots in the exposure phase was congruent with the order of the allocation of numerical characters on the vertices of invisible polygons in the rating phase. That is, participants viewed and visualized to-be-rated invisible polygons in the same spatiotemporal pattern as they did in the exposure phase (for the mere exposure effect to the spatial pattern of stimuli, see Gordon \& Holyoak, 1983, Experiment 2). Therefore, it is possible to consider that pair of dots, one of which was the same location as the dot in the preceding pair, was flashed in succession to draw the invisible polygon. The flickering dots rotated counterclockwise on the entire side of the invisible polygon two times

the mere exposure effect occurred for the spatiotemporal patterns acquired in the exposure phase. To clarify, in Experiment 2 , the order of the flashed dots was incongruent with the order of the numerical characters on the vertices of the invisible polygons. Experiment 3 was conducted to ensure that the mere exposure effect occurs for the visual representation of invisible polygons but not for spatial positions of the flashed dots. To investigate this, participants were not asked to visualize invisible polygons in the exposure phase.

Finally, Experiment 4 aimed to ascertain the role of perceptual fluency in the mere exposure effect for visual representations. To this end, we considered the theory of transferappropriate processing, which assumes that memory performance is better when encoding and retrieval processes are similar (Morris, Bransford, \& Franks, 1977). A previous study reported that transfer-appropriate processing theory was also applicable to a priming task (Franks, Bilbrey, Lien, \& McNamara, 2000). That is, a maximum priming effect was found when the same task was conducted in both the first and second phases. Given this finding, it is reasonable to assume that participants experience facilitated processing and thereby
1

$\begin{array}{llll} & & & 4 \\ & & & \\ & 5 & & \\ & & 3 & \end{array}$

Experiments 1 and 3

Fig. 2 Example of stimuli in a rating phase. In Experiments 1, 2, and 3, consecutive numerical characters were superimposed on the vertices of invisible polygons. In Experiments 1 and 3, the numerical characters were
2

54

3

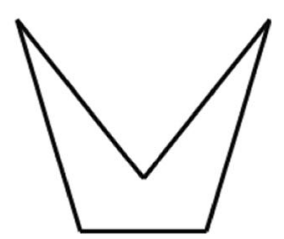

Experiment 4

presented in a counterclockwise direction, whereas in Experiment 2 they were presented in a clockwise direction. In Experiment 4, only visible polygons were presented 
feel a higher perceptual fluency when they process previously exposed stimuli in a manner consistent with earlier processing. Then, we predicted that conducting identical processing (i.e., visualizing polygons) in both the exposure and rating phases is necessary for the mere exposure effect in terms of visual representation. To assess this in Experiment 4, participants were asked to only rate their preference for visible polygons that had been repeatedly visualized in the preceding exposure phase.

\section{Experiment 1}

The aim of Experiment 1 was to investigate whether the internal representation of repeated stimuli, but not their sensory input, plays an important role in the mere exposure effect. Accordingly, participants were asked to visualize the same invisible polygons in both the exposure and the rating phases, although the stimuli presented in the exposure phase were dissimilar in sensory input to the stimuli presented in the rating phase. If the mere exposure effect is found in the experimental setting where the sensory input from stimuli is rather different between the two phases, the result would suggest that a mere exposure effect occurs for the internal representation of the invisible polygons but not for the sensory inputs themselves.

\section{Method}

Participants To determine the sample size in Experiment 1, we assumed that the effect size of the mere exposure effect is Cohen's $d=0.8$. This value corresponds to a large effect size given guidelines of Cohen (1988). The choice of the large effect size was appropriate considering that in the metaanalysis of the mere exposure effect (Bornstein, 1989), the effect size of polygons is $r=.41$, which corresponds to $d=$ 0.90 . We calculated the sample size to achieve a statistical power of approximately .90 at a significance level of $\alpha=$ .05 . The required sample size was 19 . Thus, participants were recruited so that the final sample size was greater than 19 . Twenty-four adults participated in this experiment, but three were excluded from the analysis because they became aware of the purpose of the experiment. All participants reported normal or corrected-to-normal vision.

Stimuli and apparatus All stimuli were presented on a 19inch liquid crystal display at a resolution of $1024 \times 768$ pixels. Stimulus presentation and response collection were controlled by a personal computer running Windows XP and a program developed with Hot Soup Processor (Onion Software).

To reduce the influence of preexisting variability in preference, thereby increasing the statistical power of this experiment, we generated 15 invisible polygons with five corners.
These polygons were based on those used in a previous study (Vanderplas \& Garvin, 1959). Five polygons were used in practice trials, with the remainder used in experimental trials. The polygons for experimental trials were divided into two sets, each with the approximately same mean value of preference. The maximum size of the polygons was about $7.32 \mathrm{deg}$. in width and height. A $5 \times 5$ black dot array was used for the presentation of the invisible polygons (see Fig. 1). The width and height of the dot array were 7.83 degrees, respectively, with each dot spacing in 1.83 degrees. The vertices of the invisible polygons were superimposed on the array of 25 dots.

Procedure All procedures in this and following experiments were approved by a local ethics committee of the institution to which the first author belonged. Written informed consent was obtained before the start of the experiment. To ensure that participants were unaware of the true purpose of the study, they were told that they would participate in a memory experiment. In fact, however, a memory test was not conducted. Debriefing was provided to the participants at the end of the experiment.

In the exposure phase, each trial began with the presentation of a fixation point for $500 \mathrm{~ms}$, followed by the presentation of a $5 \times 5$ dots array (see Fig. 1). After a 800 -ms interval, a pair of dots corresponding to adjacent vertices of an otherwise invisible polygon was briefly flashed, changing color to red for $800 \mathrm{~ms}$, and then returning to black for $800 \mathrm{~ms}$. Another pair of dots, one of which was at the same location as one of the dots in the preceding pair, flashed in succession to indicate the invisible polygon in a counterclockwise direction. The flashing dot sequence started from the most left vertex of the invisible polygon and then terminated when the vertices of the invisible polygon were completely rotated two times. Participants were asked to visualize and memorize the shape of invisible polygon by connecting the flashed dots with imaginary lines.

The exposure phase consisted of five practice and 25 experimental trials. In each practice trial, participants had to write down the imagined polygon after stimulus display to ensure they followed the instructions; however, on the experimental trials, they were asked not to write down the imagined polygon. In the experimental trials, each participant was assigned to one of the two sets of five polygons; the assignment was counterbalanced across participants. Each invisible polygon emerged five times in a random order, with the restriction that the same polygon never appeared on three consecutive trials. To attenuate any effect of explicit memory, the participants rested for 5 minutes before the following rating phase.

At the beginning of the rating phase, participants were told that the purpose of this phase was to collect preliminarily data for other experiments. In the rating phase, numerical characters from 1 to 5 were presented together, so that each character represented one of the five vertices of an invisible polygon in a 
counterclockwise direction (see Fig. 2). Participants were required to visualize the shape of the invisible polygon by connecting the consecutive numbers with imaginary lines in ascending order. Then, they were asked to rate their preference for the imagined polygon on a 5-point scale ( $1=$ not preferable, $5=$ preferable). Five practice and 10 experimental trials were conducted. In half of the experimental trials, the shape of to be visualized polygons was same as that of the exposure phase. In the other half of trials, these polygons were presented with a novel shape. Presentation order of these polygons was randomized across participants.

\section{Results and discussion}

Mean preference scores from the rating phase were calculated in each exposure condition for each participant (see Fig. 3). A paired $t$ test, conducted on the mean preference scores in the exposed and novel conditions, showed that mean preference scores were higher in the exposed condition than in the novel condition, $t(20)=3.48, p=.002, d=0.78$, indicating that the mere exposure effect occurred in this experimental setting. In Experiment 1, all stimuli presented in the exposure phase differed from those presented in the rating phase (Fig. 1 versus Fig. 2). Therefore, this result suggests that sensory input from superficial properties of repeated stimuli played little role in the mere exposure effect. Instead, it is plausible to infer that the mere exposure effect occurred for an internalized (cognitive) visual representation of invisible polygons.

However, it remains possible that the mere exposure effect was influenced by the spatiotemporal pattern of flashed dots and was not determined by an internalized visual representation of the invisible polygons. In Experiment 1, the order of vertices of flashed dots was identical to the progression of numerical characters. That is, participants observed the stimuli in the rating phase based on the same spatiotemporal pattern acquired in the exposure phase. Therefore, it is possible that the mere exposure effect occurred due to learning the spatiotemporal pattern of the flashed dots (Gordon \& Holyoak,

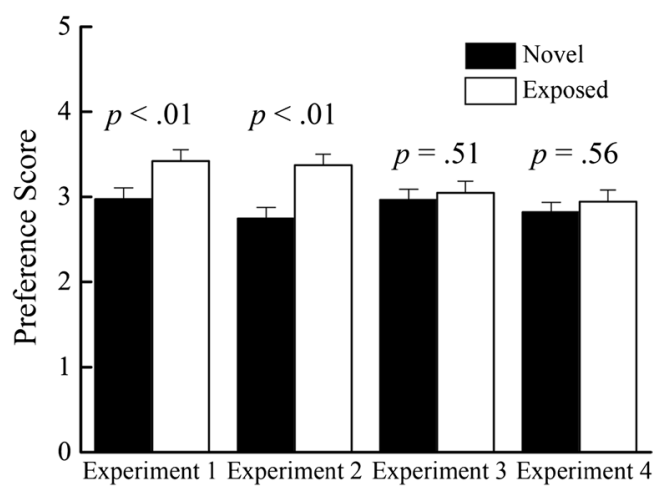

Fig. 3 Mean preference scores in all experiments. Black bars indicate results in the novel condition, and white bars indicate results in the exposed condition. Error bars show $+1 S E$
1983) and not to learning of the visual representation of the invisible polygons. We examined this possibility in Experiment 2.

\section{Experiment 2}

Experiment 2 was conducted to investigate the mere exposure effect observed in Experiment 1. Specifically, the present experiment sought to ascertain if this effect depended on spatial temporal pattern of the flashed dots (Gordon \& Holyoak, 1983) or upon an internalized visual representation of the invisible polygon. To this end, participants performed the same task as in Experiment 1, except that allocation of numerical characters was reversed. That is, the participants observed the sequence of red dots in a counterclockwise direction in the exposure phase, whereas they observed the numerical characters on the vertices of invisible polygons in a clockwise direction. In this situation, the learned spatiotemporal patterns of flashed dots should differ from patterns viewed in the rating phase. Therefore, the mere exposure effect should not be found if the mere exposure effect depends on learned spatiotemporal patterns of flashed dots from the exposure phase. In contrast, the mere exposure effect should be found if this effect occurs specifically for an internalized visual representation of invisible polygons.

\section{Method}

Participants Participants were recruited to attain a final sample size equivalent to that of Experiment 1. Twenty-two adults participated in this experiment, but one of them was excluded from the analysis due of awareness of the experimental purpose. Thus, the final sample size was 21 . None had participated in Experiment 1. All participants reported normal or corrected-to-normal vision. We calculated the statistical power based on the sample size in Experiment $2(n=21)$ and the effect size observed in Experiment 1. The obtained power was .92 , which was sufficient to detect the reliable effect of exposure.

Stimuli and procedure The stimuli and procedure were same as those in Experiment 1, with the exception that in the rating phase the numerical characters on the vertices of invisible polygons were allocated in ascending order in a clockwise direction. Therefore, the learned spatiotemporal patterns of flashed dots differed from patterns presented in the rating phase.

\section{Results and discussion}

Consistent with Experiment 1, a paired $t$ test revealed a significant effect of exposure, $t(20)=3.33, p=.003, d=0.74$, 
indicating that the mere exposure effect, evident in Experiment 1, appears also in Experiment 2. In addition, the effect sizes of Experiments 1 and 2 were similar. In summary, the mere exposure effect was found irrespective of the different observing patterns between the exposure and rating phases. This finding negates the possibility that the mere exposure effect occurred for the learned spatiotemporal pattern of flashed dots in the exposure phase.

The result of Experiment 2 also denied the involvement of the fluency arising from motor systems (i.e., motor fluency). For example, Yang, Gallo, and Beilock (2009) reported that association with a fluent motor action influences the recognition of stimuli. Given that repetition of the same eyemovement patterns increases motor fluency, it is possible that such motor fluency is misattributed to the preference of stimuli, as reported in research on perceptual fluency (Bornstein \& D’Agostino, 1992, 1994; Winkielman \& Cacioppo, 2001). In Experiment 2, however, the mere exposure effect was found despite differences in eye movement patterns between the exposure and rating phases. That is, the mere exposure effect was obtained even in the situation where the motor fluency was considered to be low. Thus, the result of Experiment 2 was inconsistent with the account based on the motor fluency.

Although results of Experiments 1 and 2 provide support for the mere exposure effect based on internally generated visual representations, two other interpretations of such findings must be addressed. One interpretation posits that the mere exposure effect occurred for visual representation of imaginary polygons. Specifically, it is possible that the mere exposure effect occurred for the imaginary shape of the polygons generated in the exposure phase. The other interpretation holds that the mere exposure effect occurs for the spatial representations of dot positions (Gordon \& Holyoak, 1983). That is, it is possible that in the exposure phase participants learned only the spatial positions of flashed dots without visualizing the contours of the invisible polygons. In this case, the mere exposure effect is attributed to the spatial representation of the dot positions. In order to dissociate these two interpretations, people must be capable of memorizing the spatial positions of the flashed dots without visualizing the shape of the imaginary polygons. This possibility is examined in Experiment 3.

\section{Experiment 3}

Experiment 3 aimed to clarify whether the mere exposure effect occurs for the visual representation of invisible polygons or for the spatial representation of dot positions (Gordon \& Holyoak, 1983). To this end, participants were required to memorize the positions of flashed dots without visualizing invisible polygons in an exposure phase. However, in the following rating task, they were told to visualize the shape of the invisible polygons. If visual representations of invisible polygons play a significant role in the mere exposure effect observed in Experiments 1 and 2, the mere exposure effect should disappear (or attenuate) in Experiment 3 because the visual representation of invisible polygons cannot be constructed in the exposure phase of this experiment.

\section{Method}

Participants To determine the sample size in Experiment 3, we integrated the effect sizes in Experiments 1 and 2 using a fixed effect model of meta-analysis, $d_{\text {Exp.1 \& } 2}=0.76,95 \%$ confidence interval (CI) $[0.42,1.10]$. Based on the integrated effect size, we calculated the sample size to achieve a statistical power of approximately .90 at a significance level of $\alpha=$ .05 . The required sample size was 21 . Thereafter, 21 adults were recruited for this experiment. None had participated in Experiments 1 and 2. All participants reported normal or corrected-to-normal vision.

Stimuli and procedure The stimuli and procedure were identical to those of Experiment 1, except that in the exposure phase participants were not explicitly told to visualize the shape of invisible polygon as they memorized the spatial positions of the flashed dots. That is, participants were not told that flashing the pair of dots five times constructed one invisible polygon. Instead, they were told that flashing the pair of dots five times constructed one spatial array of dots.

\section{Results and discussion}

As in Experiment 1, a paired $t$ test was conducted on mean preference scores. In contrast to Experiments 1 and 2, the $t$ test failed to reach significance, $t(20)=0.66, p=.51, d=0.15$. Furthermore, the effect size was considerably smaller than that in Experiment $1(d=0.78)$ and in Experiment $2(d=0.74)$. To support the absence of significance, we calculated the Bayes factor (BF) for the null hypothesis (i.e., equal population means between the exposed and novel conditions) against the two-sided alternative hypothesis using JASP software (Wagenmakers et al., 2017) with a default Cauchy prior width of $0.707(\mathrm{BF}=3.61)$. This Bayes factor value indicates that the observed data were 3.61 times more likely to occur under the null hypothesis than under the alternative hypothesis, which provided positive evidence that the null hypothesis was supported (Kass \& Raftery, 1995). These results indicate that the mere exposure effect was not observed in Experiment 3. The absence of the mere exposure effect could not be attributed to the insufficient statistical power because this experiment had a sufficient statistical power (.90). Instead, these results suggest that the disturbance of visualizing invisible polygons in the exposure phase attenuates the mere exposure effect. From these results, we infer that visual representation of the imagined polygons, but not the spatial representations 
of dot positions, play an important role in the mere exposure effect observed in Experiments 1 and 2.

\section{Experiment 4}

The results of Experiments 1, 2, and 3 showed that the mere exposure effect occurred when participants visualized the shape of invisible polygons in both the exposure and rating phases, whereas it disappeared when participants visualized the shape of the invisible polygons only in the rating phase. From these results, we consider that the mere exposure effect can be found only when the same processing (i.e., visualizing polygons) is conducted in both the exposure and rating phases. Specifically, it is possible that the mere exposure effect for the visual representation of invisible polygons occurs only when both exposure and rating tasks require participants to visualize the shape of these polygons. To investigate this possibility, in Experiment 4, only visible polygons were presented in the rating phase. In this situation, participants had no need to visualize the shape of invisible polygons in the rating phase. Therefore, it is reasonable to assume that processing conducted in the rating phase is different from that conducted in the exposure phase. Consequently, if conducting the same processing in both the exposure and rating phases plays an important role in the mere exposure effect, then it is likely that the mere exposure effect should disappear in Experiment 4.

\section{Method}

Participants Based on the power analysis in Experiment 3, we recruited participants to achieve a final sample size of at least 21 participants. Twenty-four adults participated in this experiment, but three participants were excluded from analysis because they became aware of the purpose of the experiment. None participated in the previous experiments. All participants reported normal or corrected-to-normal vision.

Stimuli and procedure The stimuli and procedure were identical to those of Experiment 1, except that to-be-rated polygons were actually presented in the rating phase. Thus, participants were required to rate the preference of the visible polygons but not the visual images of invisible polygons.

\section{Results and discussion}

As in Experiment 1, a paired $t$ test was conducted on mean preference scores. In contrast to Experiments 1 and 2, this $t$ test failed to show a significant effect of exposure, $t(20)=0.60, p=$ $.56, d=0.13$. Additionally, a Bayes factor calculated similar to Experiment 3 provided positive evidence that the null hypothesis was supported $(\mathrm{BF}=3.74)$. These results indicate that the mere exposure effect was not observed in Experiment 4.
Because this experiment had relatively high statistical power, absence of the mere exposure effect suggests that different processing between the exposure and rating phases reduced the mere exposure effect, even though repeatedly visualized polygons were actually presented in the rating phase.

\section{General discussion}

The aim of the present study was to clarify whether the sensory inputs from repeated stimuli are necessary for the mere exposure effect. For this purpose, we developed a novel experimental method that allowed us to investigate the existence of mere exposure for the visual representation of invisible polygons. The results of Experiment 1 showed the mere exposure effect occurred when participants visualized the shape of the same invisible polygon in both the exposure and rating phases, despite the fact that sensory input from repeated stimuli was rather different from sensory input during the rating phase. Experiment 2 confirmed that the mere exposure effect was not impaired when viewing patterns of repeating stimuli in the exposure phase differed from those of the rating phase. In Experiment 3, the mere exposure effect was eliminated when participants were not asked to visualize the shape of the invisible polygons in the exposure phase. In Experiment 4 , the mere exposure effect for the imagined polygons was not found when participants evaluated the preference for the visible polygons that had been repeatedly imagined in the exposure phase.

The most important finding from the present study is that the sensory inputs from repeated stimuli apparently play a minor role in the mere exposure effect. Specifically, we demonstrated that the mere exposure effect occurred even though repeated stimuli in the exposure phase were rather different from those presented in the rating phase as long as participants visualized identical polygons in both the exposure and rating phases (Experiments 1 and 2). This finding contradicts the naïve view of the mere exposure effect that repeatedly "presented" stimuli are evaluated more positively than novel stimuli (Zajonc, 1968). Instead, the results of the present study suggest that repeatedly "constructed" visual representation of stimuli (in this study, visual images) plays an important role in the mere exposure effect. This is consistent with other recent findings that the mere exposure effect occurred for the internal representation modulated by top-down factors but not for repeated stimuli themselves (Craver-Lemley \& Bornstein, 2006; Yagi et al., 2009). However, these studies did not clarify whether the sensory input from repeated stimuli is necessary for the mere exposure effect due to the development of internal representations in the exposure phase because the exposure stimuli were contained, or were similar to those, in the rating phase. In contrast to these studies, our study demonstrated that the mere exposure effect occurred even when the 
stimulus display in the exposure phase was physically dissimilar to the stimulus display in the rating phase. This suggests that the sensory input from repeated external stimulation is not a significant factor in development of a mere exposure effect based on internal representations. The finding that sensory inputs had a minor role in the mere exposure effect resembles results reported by Monahan et al. (2000), who reported that repeated presentation of stimuli increases positive affect even to novel stimuli (i.e., previously not presented stimuli). However, in contrast to Monahan et al. (2000), we demonstrated the significance of internal representations as well as the lack of significance of sensory inputs in identical experimental situations. In this sense, the present study further advanced an understanding of the relative importance of these factors in the mere exposure effect.

To explain the results of the present study, we must consider another factor, namely, the consistency of processing between the exposure and rating phases. In the present study, the mere exposure effect was found when participants visualized the holistic shape of invisible polygons in both the exposure and rating phases (Experiments 1 and 2), whereas it was not evident when participants visualized the shape of the invisible polygons only in the exposure phase (Experiment 4) or only in the rating phase (Experiment 3). From these results, we consider that the mere exposure effect is facilitated when the same processing (i.e., imaging polygons) is required in both the exposure and rating phases. To pursue this interpretation, we conducted a smallscale meta-analysis following the recommendation of Cumming (2012). As already described, the integrated effect size between Experiments 1 and 2 is $d_{\text {Exp. } 1 \& 2}=0.76,95 \%$ CI $[0.42,1.10]$. On the other hand, the integrated effect size between Experiments 3 and 4 is $d_{\text {Exp. } 3 \& 4}=0.15,95 \%$ CI $[-0.16,0.45]$. Note that the $95 \%$ CI of $d_{\text {Exp. } 3 \text { \& } 4}$ largely overlapped with $d=0$ (i.e., the absence of the mere exposure effect). This indicates that the mere exposure effect did not occur when participants visualized the shape of the invisible polygons in either the exposure or the rating phase, which is also supported by the Bayes factor calculated for the results of Experiments 3 and $4(\mathrm{BF}=4.20)$. Furthermore, to compare $d_{\mathrm{Exp} .1} \& 2$ with $d_{\text {Exp.3 \& } 4 \text {, we conducted an analysis of heterogeneity, indicating }}$ that the two effect sizes were not derived from the same population, $Q(1)=7.02, p=.008, I^{2}=86 \%$. That is, the mere exposure effect was greater when the same processing was conducted in both the exposure and rating phases (Experiments 1 and 2) than when the same processing was not conducted in both phases (Experiments 3 and 4). ${ }^{1}$ In summary, these analyses suggest that, in addition to the construction of the visual representation, the consistency of processing between the exposure

\footnotetext{
${ }^{1}$ We also conducted an analysis of heterogeneity of three effect sizes $\left(d_{\text {Exp.1 \& }}\right.$ ${ }_{2}, d_{\text {Exp.3 } 3}$, and $d_{\text {Exp.4 }}$ ), indicating that these effect sizes were not derived from the same population, $Q(2)=7.02, p=.03, I^{2}=72 \%$. The results suggest that the integrated effect size between Experiments 1 and 2 is different from each effect size in Experiments 3 and 4.
}

and rating phases also plays a significant role in the visual mere exposure effect.

The importance of the consistency of processing could be explained by enhancement of perceptual fluency (Bornstein \& D'Agostino, 1992, 1994). Consistent with the theory of transfer-appropriate processing (Morris et al., 1977), Franks et al. (2000) found a maximum priming effect when the same task was conducted in both the first and second phases. Given this finding, it is reasonable to consider that participants' experiences facilitated processing and they thereby felt a higher perceptual fluency when they processed previously exposed stimuli in a manner consistent with earlier processing. In the rating phase of Experiments 1 and 2, participants visualized the shape of invisible polygons as they did in the preceding exposure phase. This consistency of the processing leads to the enhanced perceptual fluency to repeatedly imagined polygons, which consequently could induce the liking of these polygons. Thus, results of the present study provide further evidence for the view of perceptual fluency (Bornstein \& D'Agostino, 1992, 1994; Winkielman \& Cacioppo, 2001).

One may think that the mere exposure effect occurred due to the learning of the spatiotemporal patterns or spatial positions of flashed dots. In the exposure phase of Experiment 1, participants were repeatedly presented with specific moving patterns of flashed dots and in the rating phase participants observed an array of numerical characters based on the same spatiotemporal patterns learned in the exposure phase. Given that the mere exposure effect is associated with learning of various regularities such as artificial grammar or spatial patterns (Gordon \& Holyoak, 1983), it is plausible that acquisition of the spatiotemporal patterns in the exposure phase may lead to the mere exposure effect. However, results of Experiment 2 dispute this explanation. In that experiment the mere exposure effect appears even in a situation where putatively learned spatiotemporal patterns of flashed dots in the exposure phase differed from the presented patterns of the numerical array in the rating phase. Consequently, such results cannot be attributed to earlier learning of the spatiotemporal patterns of flashed dots. Similarly, it is unlikely that the mere exposure effect occurred because of the learning of the spatial positions of flashed dots, because the instruction to learn the positions of flashed dots actually attenuated the mere exposure effect in Experiment 3. In summary, conversing evidence suggests that the mere exposure effect occurred for the visual representation of invisible polygons.

The absence of the mere exposure effect in Experiment 4, where participants evaluated the preference for the visible polygons that were repeatedly visualized, is consistent with the theory of transfer-appropriate processing (Morris et al., 1977). According to this theory, memory performance is sensitive to a difference in processing strategy between a learning phase and a memory test phase. Considering that some form of memory is involved in the mere exposure effect (e.g., Seamon et al., 1995), 
it is reasonable to consider that the mere exposure effect is also sensitive to a difference in processing strategy between an exposure task and a rating task. Consistent with this interpretation, the mere exposure effect was absent in Experiment 4 of the present study. In this experiment, the processing conducted in the exposure phase (i.e., visualizing polygons) was not required in the rating phase. However, the mere exposure effect was found in Experiments 1 and 2, where the same processing was conducted in both the exposure and the rating phases. Although this study does not provide conclusive evidence for the absence of the mere exposure effect in Experiment 4, it is reasonable to hypothesize that changing the processing strategy between the exposure and rating phases diminishes the mere exposure effect.

It is worth discussing the results of the present study from the viewpoints of other hypotheses. One is the affective theory of the mere exposure effect, which assumes that repeated exposure of stimuli increases positive affect to those stimuli without involvement of cognitive process (Zajonc, 1980). This theory especially emphasizes that the mere exposure could occur without explicit recognition of repeated stimuli. Although we cannot speak to the necessity of recognition memory, the present study at least suggests that in some situations cognitive processes, such as constructing visual representation, plays an important role in the mere exposure effect. In fact, the different results between Experiments 1 and 4 cannot be explained without assuming a difference in cognitive process required in the rating phase. We do not suggest that cognitive process is always critical for the mere exposure effect, because previous studies have shown that this effect occurs with minimal intervening cognitive activity (e.g., Elliott \& Dolan, 1998; Kunst-Wilson \& Zajonc, 1980). Instead, it is important to clarify the conditions where cognitive process has an influence on the mere exposure effect (Craver-Lemley \& Bornstein, 2006; Yagi et al., 2009).

Another hypothesis to be considered assumes that reduced alertness and tension for repeated stimuli are involved in the mere exposure effect (Monahan et al., 2000; see also Zajonc, 2001). Given this hypothesis, it is possible that repeatedly imaging the shape of the invisible polygons reduces alertness and tension for the shape of the polygons, and then increases the preference for them. Thus, this hypothesis is consistent with the presence of the mere exposure effect in Experiments 1 and 2. However, it appears to be inconsistent with the absence of the mere exposure effect in Experiment 4. It is less reasonable to consider that repeatedly constructing the visual image of polygons does not reduce the tension for the actually presented polygons having the same shape as polygons imagined in the exposure phase. Although the results of the present study might favor the perceptual fluency theory (Bornstein \& D'Agostino, 1992, 1994; Winkielman \& Cacioppo, 2001), it is beyond the scope of the present study to conclude which of these hypotheses holds more validity as an explanation for the mere exposure effect; further studies are needed.
The results of the present study cannot be explained by a recognition memory bias. That is, a bias raises the possibility that participants simply provided higher values of preference for explicitly recognized polygons. However, if this were the case, then the mere exposure effect should have been observed in Experiment 4. Furthermore, many previous studies of the mere exposure effect have reported that explicit recognition of the exposed stimuli was independent from the mere exposure effect (e.g., Matlin, 1971; Moreland \& Zajonc, 1977; Yagi et al., 2009; but see Brooks \& Watkins, 1989). That is, these studies showed no correlation between the recognition memory and mere exposure effect.

We do not suggest that the mere exposure effect always occurs for internal representations modulated by top-down mechanism. As indicated by previous and present studies, top-down factors such as selective attention and visual image had an influence on the mere exposure effect for supraliminal stimuli (Craver-Lemley \& Bornstein, 2006; Yagi et al., 2009). On the other hand, these factors may be less effective for subliminal stimuli when participants have difficulty modulating the processing of the subliminal stimuli via the top-down factors (Jung \& Chong, 2014). In such situations, the representation of subliminal stimuli may be determined largely by a bottom-up factor such as the sensory input from the stimuli; if so, then the resulting mere exposure effect should reflect the actual stimuli presented.

In conclusion, the present study strengthens the relevance of internal representations in the mere exposure effect (Craver-Lemley \& Bornstein, 2006; Yagi et al., 2009) by demonstrating that the sensory input from repeated stimuli is not a necessary factor to the mere exposure effect. Furthermore, we suggest that the construction of visual representation, the consistency of processing between the exposure and rating phases is also necessary condition for the mere exposure effect. Future studies should consider these findings to elucidate the mechanism of the mere exposure effect.

Author note This work was supported by a Grant-in-aid (JSPS KAKENHI Grant Nos. 20530657, 15K00211, and 26870751) from the Japan Society for the Promotion of Science.

We would like to thank to Prof. Tadashi Kikuchi. He is an important collaborator on this study. However, we are saddened to report that he passed away prior to the completion of this article.

\section{References}

Bornstein, R. F. (1989). Exposure and affect: Overview and meta-analysis of research, 1968-1987. Psychological Bulletin, 106(2), 265-289. doi:https://doi.org/10.1037/0033-2909.106.2.265

Bornstein, R. F., \& D'Agostino, P. R. (1992). Stimulus recognition and the mere exposure effect. Journal of Personality and Social Psychology, 63(4), 545-552. doi:https://doi.org/10.1037/00223514.63.4.545 
Bornstein, R. F., \& D'Agostino, P. R. (1994). The Attribution and discounting of perceptual fluency: Preliminary tests of a perceptual fluency/attributional model of the mere exposure effect. Social Cognition, 12(2), 103-128. doi:https://doi.org/10.1521/soco.1994. 12.2.103

Brooks, J. O., \& Watkins, M. J. (1989). Recognition memory and the mere exposure effect. Journal of Experimental Psychology: Learning, Memory, and Cognition, 15(5), 968-976. doi:https://doi. org/10.1037/0278-7393.15.5.968

Cohen, J. (1988). Statistical power analysis for the behavioral science (2nd ed.). Hillsdale: Erlbaum.

Craver-Lemley, C., \& Bornstein, R. F. (2006). Self-generated visual imagery alters the mere exposure effect. Psychonomic Bulletin \& Review, 13(6), 1056-1060. doi:https://doi.org/10.3758/ BF03213925

Cumming, G. (2012). Understanding the new statistics: Effect sizes, confidence intervals, and meta-analysis. New York: Routledge.

Elliott, R., \& Dolan, R. J. (1998). Neural response during preference and memory judgments for subliminally presented stimuli: A functional neuroimaging study. Journal of Neuroscience, 18(12), 4697-4704.

Franks, J. J., Bilbrey, C. W., Lien, K. G., \& McNamara, T. P. (2000). Transfer-appropriate processing (TAP) and repetition priming. Memory \& Cognition, 28(7), 1140-1151. doi:https://doi.org/10. 3758/BF03211815

Gordon, P. C., \& Holyoak, K. J. (1983). Implicit learning and generalization of the "mere exposure" effect. Journal of Personality and Social Psychology, 45(3), 492-500. doi:https://doi.org/10.1037/ 0022-3514.45.3.492

Jung, Y., \& Chong, S. C. (2014). Effects of attention on visible and invisible adapters. Perception, 43(6), 549-568. doi:https://doi.org/ $10.1068 / \mathrm{p} 7660$

Kass, R. E., \& Raftery, A. E. (1995). Bayes factors. Journal of the American Statistical Association, 90, 773-795. doi:https://doi.org/ 10.1080/01621459.1995.10476572

Kramer, R. S. S., \& Parkinson, B. (2005). Generalization of mere exposure to faces viewed from different horizontal angles. Social Cognition, 23(2), 125-136. doi:https://doi.org/10.1521/soco.23.2. 125.65625

Kunst-Wilson, W., \& Zajonc, R. (1980). Affective discrimination of stimuli that cannot be recognized. Science, 207(4430), 557-558. doi: https://doi.org/10.1126/science.7352271

Mandler, G., Nakamura, Y., \& Van Zandt, B. J. (1987). Nonspecific effects of exposure on stimuli that cannot be recognized. Journal of Experimental Psychology: Learning, Memory, and Cognition, 13(4), 646-648. doi:https://doi.org/10.1037/0278-7393.13.4.646

Matlin, M. W. (1971). Response competition, recognition, and affect. Journal of Personality and Social Psychology, 19(3), 295-300. doi:https://doi.org/10.1037/h0031352

Monahan, J. L., Murphy, S. T., \& Zajonc, R. B. (2000). Subliminal mere exposure: Specific, general, and diffuse effects. Psychological Science, 11(6), 462-466. doi:https://doi.org/10.1111/1467-9280. 00289

Moreland, R. L., \& Zajonc, R. (1977). Is stimulus recognition a necessary condition for the occurrence of exposure effects? Journal of Personality and Social Psychology, 35(4), 191-199. doi:https:// doi.org/10.1037/0022-3514.35.4.191

Morris, C. D., Bransford, J. D., \& Franks, J. J. (1977). Levels of processing versus transfer appropriate processing. Journal of Verbal Learning and Verbal Behavior, 16, 519-533. doi:https://doi.org/ 10.1016/S0022-5371(77)80016-9
Reber, R., Schwarz, N., \& Winkielman, P. (2004). Processing fluency and aesthetic pleasure: Is beauty in the perceiver's processing experience? Personality and Social Psychology Review, 8(4), 364-382. doi:https://doi.org/10.1207/s15327957pspr0804_3

Rhodes, G., Halberstadt, J., \& Brajkovich, G. (2001). Generalization of mere exposure effects to averaged composite faces. Social Cognition, 19(1), 57-70. doi:https://doi.org/10.1521/soco.19.1.57. 18961

Seamon, J. G., Brody, N., \& Kauff, D. M. (1983). Affective discrimination of stimuli that are not recognized: Effects of shadowing, masking, and cerebral laterality. Journal of Experimental Psychology: Learning, Memory, and Cognition, 9(3), 544-555. doi:https://doi.org/10.1037/0278-7393.9.3.544

Seamon, J. G., Ganor-Stern, D., Crowley, M. J., Wilson, S. M., Weber, W. J., O'Rourke, C. M., \& Mahoney, J. K. (1997). A mere exposure effect for transformed three-dimensional objects: Effects of reflection, size, or color changes on affect and recognition. Memory \& Cognition, 25(3), 367-374. doi:https://doi.org/10.3758/ BF03211292

Seamon, J. G., Williams, P. C., Crowley, M. J., Kim, I. J., Langer, S. A., Orne, P. J., \& Wishengrad, D. L. (1995). The mere exposure effect is based on implicit memory: Effects of stimulus type, encoding conditions, and number of exposures on recognition and affect judgments. Journal of Experimental Psychology: Learning, Memory, and Cognition, 21(3), 711-721. doi:https://doi.org/10.1037/02787393.21.3.711

Topolinski, S., \& Strack, F. (2009). The architecture of intuition: Fluency and affect determine intuitive judgments of semantic and visual coherence and judgments of grammaticality in artificial grammar learning. Journal of Experimental Psychology: General, 138(1), 39-63. doi:https://doi.org/10.1037/a0014678

Vanderplas, J. M., \& Garvin, E. A. (1959). The association value of random shapes. Journal of Experimental Psychology, 57(3), 147154. doi:https://doi.org/10.1037/h0048723

Wagenmakers, E.-J., Love, J., Marsman, M., Jamil, T., Ly, A., Verhagen, J., Selker, R., ... Morey, R. D. (2017). Bayesian inference for psychology: Part 2. Example applications with JASP. Psychonomic Bulletin \& Review. doi:https://doi.org/10.3758/s13423-017-1323-7

Winkielman, P., \& Cacioppo, J. T. (2001). Mind at ease puts a smile on the face: Psychophysiological evidence that processing facilitation elicits positive affect. Journal of Personality and Social Psychology, 81(6), 989-1000. doi:https://doi.org/10.1037//0022-3514.81.6.989

Yagi, Y., Ikoma, S., \& Kikuchi, T. (2009). Attentional modulation of the mere exposure effect. Journal of Experimental Psychology: Learning, Memory, and Cognition, 35(6), 1403-1410. doi:https:// doi.org/10.1037/a0017396

Yang, S., Gallo, D. A., \& Beilock, S. L. (2009). Embodied memory judgements: A case of motor fluency. Journal of Experimental Psychology, Learning, Memory, and Cognition, 35(5), 1359-1365. doi:https://doi.org/10.1037/a0016547

Zajonc, R. B. (1968). Attitudinal effects of mere exposure. Journal of Personality and Social Psychology, 9(2, Pt. 2), 1-27. doi:https:// doi.org/10.1037/h0025848

Zajonc, R. B. (1980). Feeling and thinking: Preferences need no inference. American Psychologist, 35(2), 151-175. doi:https://doi.org/ 10.1037/0003-066X.35.2.151

Zajonc, R. B. (2001). Mere exposure: A gateway to the subliminal. Current Directions in Psychological Science, 10(6), 224-228. doi: https://doi.org/10.1111/1467-8721.00154 